\title{
Active Optical Lattice Filters
}

\author{
L. Roberts Hunt, ${ }^{1}$ Vishnupriya Govindan, ${ }^{1}$ Issa Panahi, ${ }^{1}$ Jian Tong, ${ }^{1}$ Govind Kannan, ${ }^{1}$ \\ Duncan L. MacFarlane, ${ }^{1}$ and Gary Evans ${ }^{2}$ \\ ${ }^{1}$ Erik Jonsson School of Engineering and Computer Science, The University of Texas at Dallas, Richardson, TX 75083-0688, USA \\ Emails: hunt@utdallas.edu,vishnupriyagovindan@hotmail.com,issa.panahi@utdallas.edu, jxt021000@utdallas.edu, \\ govind@student.utdallas.edu,dlm@utdallas.edu \\ ${ }^{2}$ School of Engineering and Applied Science, Southern Methodist University, Dallas, TX 75275-0335, USA \\ Email: gae@engr.smu.edu
}

Received 26 March 2004; Revised 20 October 2004

Optical lattice filter structures including gains are introduced and analyzed. The photonic realization of the active, adaptive lattice filter is described. The algorithms which map between gains space and filter coefficients space are presented and studied. The sensitivities of filter parameters with respect to gains are derived and calculated. An example which is relevant to adaptive signal processing is also provided.

Keywords and phrases: active, adaptive, filter, optical, lattice.

\section{INTRODUCTION}

Dowling and MacFarlane [1, 2, 3] viewed a lightwave lattice filter design using multistage etalons and resonators as a discrete-time linear system. As such they discussed the behavior of the system in terms of the transmission and reflection transfer functions. Given a desired transfer function denominator for an all-pole system in transmission they developed a layer-peeling algorithm to determine the appropriate reflection coefficients. The reflection transfer function has this same denominator polynomial. They proved that any denominator polynomial, and hence any autoregressive (AR) transfer function in transmission, can be generated by proper choice of the reflection coefficients. Transmission coefficients as well as reflection coefficients can be chosen, but these transmission coefficients do not affect the denominator polynomial. If an autoregressive moving average (ARMA) transfer function is desired in transmission, then it must first be approximated by an AR transfer function of higher order, and a higher-order lattice filter be designed.

The problem with the above optical lattice filter is that it is a passive device, and once it is built with a given set of reflection (also transmission) coefficients, its filtering characteristics cannot be changed. Hence this precludes programming in the field to change the filter as needed. Moreover,

This is an open access article distributed under the Creative Commons Attribution License, which permits unrestricted use, distribution, and reproduction in any medium, provided the original work is properly cited. from the point of view of signal processing, the entire set of applications derived from adaptive signal processing is not accessible. Adaptive signal processing is used in communications, control, radar, sonar, seismology, biomedical, target tracking, and so forth [4]. Adaptation is needed if the statistics of a signal are unknown or if a system is time varying (nonstationary). Finite impulse response (FIR) filters have dominated the field, but infinite impulse response (IIR) filters can often provide a system of much lower order [5].

To add adjustment to these optical lattice filters we propose to add gains to each stage. These gains can be changed as needed and also serve to make our filters active instead of passive. Suppose we are given a target all-pole transfer function for transmission whose order is the same as the order (number of stages) of our optical filter. Once the reflection coefficients (by layer peeling) and transmission coefficients are determined and the device is built, then we tune the device by changing the gains. The question of interest is what all-pole transfer functions (or denominator polynomials) can we achieve by changing the gains? Certainly when the gains are all unity we have the denominator polynomial of the all-pole transfer function that we started. From the point of tunable signal processing it would be desirable to generate all "nearby" polynomials of the same degree.

It is interesting to note that after fixing these reflection and transmission coefficients, it is not possible to use the gains to generate an arbitrary all-pole transfer function in transmission. We present an example to illustrate this point. However, if we consider all gains as one when the reflection and transmission coefficients are fixed, then there is an open 
neighborhood $U$ of these ones in gains space and an open neighborhood $V$ of the corresponding denominator polynomial coefficients in polynomial coefficients space, so that for each polynomial with coefficients in $V$ there is a unique set of gains in $U$ that produce that polynomial. All that needs to be checked is that the Jacobian matrix of the map from the gains (actually gains squared) to the polynomial coefficients is nonsingular when the gains are all one. If this matrix is nonsingular it is natural to solve for the gains as functions of the coefficients using the standard Newton-Raphson method $[6,7]$. The equations expressing the relationship of the polynomial coefficients and the gains are easily generated and have a high degree of symmetry. Hence we have a procedure that will allow us to calculate all nearby (in terms of polynomial coefficients) denominator polynomials for our transfer functions. Thus we can use gains to adapt our filter. Of course we are only interested in stable filters when applications are considered.

For the map from the gains to the denominator polynomial coefficients we stress the case where the gains are all one. However, for a given denominator polynomial there may be other sets of gains that yield the same polynomial coefficients. If for such a point in gain space, the Jacobian matrix of the gains to polynomial coefficients map is nonsingular, then there is an open neighborhood $U^{*}$ of the gain point and an open neighborhood $V^{*}$ of the corresponding polynomial coefficient point in polynomial coefficient space on which the map is one-to-one and onto. Of course $U$ and $U^{*}$ cannot intersect, so we can adapt in the set $U$ (or $U^{*}$ ) without disturbing the other set.

The main purpose of this paper is to provide design tools for active lattice filters that contain active gain elements. This discussion is particularly relevant to an optical architecture that is currently under early development. This photonic realization of the active lattice filter is described in Section 2 of this paper. In particular we advocate a semiconductor laser amplifier structure in which coupling between gain and delay stages is accomplished by surface gratings [8]. While there is a rich tradition of active electronic filters, optical filters with gain are not generally used. Gain elements allow filters with high-quality factors, and filters with programmable transfer functions. Further, gain elements are essential to providing an architecture that may be scaled to reasonable sophistication.

In Section 3 of this paper we discuss the DowlingMacFarlane optical lattice filter and the layer-peeling procedure for computing the reflection coefficients. We indicate how gains are added to their structure. We then compute the transfer function denominator polynomials as functions of the gains assuming that all reflection and transmission coefficients have been fixed. In Section 4 we provide an example showing that an arbitrary transmission all-pole denominator cannot be generated using gains once the reflection and transmission coefficients have been chosen. Here we work with a two-stage filter and a second-order transfer function denominator polynomial. We generate the map from twodimensional gains space to two-dimensional polynomial coefficients space. For given polynomial coefficients this map can be represented by two curves in gains space and there are three possible outcomes: transversal intersection where the Jacobian matrix is nonsingular, no intersection and no solution, and tangential intersection where the Jacobian matrix is singular. It is the first case that is of interest to us. It is important to understand that we start with a given polynomial and fix the reflection coefficients so that we have this case if the Jacobian matrix is nonsingular when all gains are unity. Then all "nearby polynomials" can be implemented by a proper choice of gains.

Section 5 contains a development of the map from gains (squared) space to denominator polynomial coefficient space in the general case. Then we state and prove our main result using the inverse function theorem [9]. The assumption on the nonsingularity of the Jacobian matrix is the exact condition needed to employ the Newton-Raphson algorithm to solve our equations for the gains. We return to the example in Section 4 for the nonsingular case, vary the coefficients of the polynomial, and show the computations of the gains. We also provide an example that is relevant to adaptive signal processing, assuming that a feedback loop may be implemented that is fast enough to respond to a changing environment. We start with an output response of an AR system. Next we identify the all-pole transfer function and determine the appropriate reflection coefficients (through layer peeling), which we fix in our lattice filter. Then we allow the time response to change, identify the corresponding denominator polynomials for the new transfer functions, and compute the gains to deliver those polynomials. Here the time response could be replaced by a frequency response or by autocorrelations. In Section 6 we derive and calculate the sensitivities of filter parameters with respect to gains. Section 7 contains our conclusions and a discussion of future research.

\section{A REALIZATION ARCHITECTURE}

The optical gains that are modeled in this paper may be realized in a number of ways. For example, it is possible to imagine using erbium-doped fibers or waveguides separated by fiber Bragg gratings or an equivalent coupling interface. The passive version of this fiber based-filter architecture is very nicely described in the classic paper by Moslehi et al. [10]. The active fiber filter with gain would have particular interest for fiber sensors and instrumentation [11]. In the interests of faster updating speeds and adaptive operation we are envisioning a semiconductor laser amplifier structure in which coupling between gain and delay stages is accomplished by surface gratings. This architecture also offers faster clock frequencies, higher efficiencies and integrated manufacture advantages.

In Figure 1 is shown a schematic of the active optical lattice filter that uses semiconductor laser amplifier stages to provide gain and delays. By way of example, the figure shows a two-stage active lattice filter with two gain/delay regions and three interstage couplers. The figure is drawn in side view and shows a substrate with an epitaxial-grown quantum well active region. On the surface of this substrate are two electrodes through which injection current may enter the 


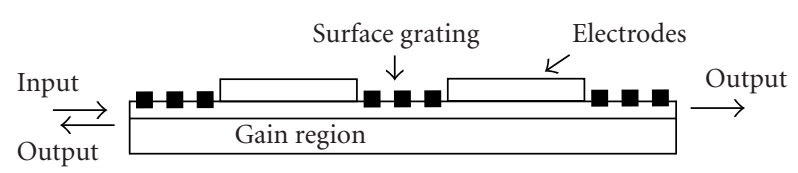

FIGURE 1: Block diagram of an active lattice filter based on surface grating interstage couplers and semiconductor optical amplifiers.

quantum well. The filter stage gain is controlled by the injection current to the individually addressable gains in the amplifying regions. These regions also provide the phase delays that lead to filter responses.

Also on the surface of the substrate are three grating couplers that provide transmission and reflection of optical signals between the two stages under the two electrodes. These gratings may be written by holographic lithography $[8,12]$ or by focused ion beam micromachining.

In operation, an optical signal is injected into the heterojunction of the structure where it undergoes gain and delay. At the grating couplers part of the signal is reflected and part is transmitted. Each of these components combines with signals from the adjacent stage, and this delay and mix action is the same as in coupled etalons, or in thin film filters. The additional presence of gain provides advantages in filter performance including a tunable response.

In practice, the semiconductor optical amplifier may suffer from two impairments: saturation and phase distortion. Since the theory presented herein applies to linear, timeinvariant (LTI) systems, either impairment will render the results presented in this paper an approximation valid over a finite region of operation. Since the filter will be operated in a stable region, well below lasing threshold, the overall gains needed for useful filters will be small, and will vary over a limited range. Hence the linearity condition will often be justified. Similarly, it may also be argued that in this operating region, the injected current will not substantially impact the refractive index of the inversion region, particularly if the effective gain length is kept short and the epitaxy is properly designed. An additional possibility for adjusting and maintaining the correct phase is to add an additional phase control section to the filter as is done in many tunable lasers so as to allow an additional adjustment for phase control.

\section{DOWLING-MACFARLANE FILTER}

In 1944 MacFarlane and Dowling studied the analysis and design of purely passive coupled Fabry-Perot etalons and thin film filters using digital signal processing techniques $[1,2]$. This approach allowed certain design optimizations, and brought an ease of use that proved helpful to electrical engineers working in the photonic telecommunications industry over the last decade. In Figure 2 is shown a $z$-transformbased block diagram for an exemplary three-stage photonic lattice filter. The interfaces are characterized by reflection and transmission coefficients, and the signal transit time between interfaces is described by a delay block. Considering the realization architecture discussed above, we follow the notation of [2]. For a lossless interface, the field reflection and transmission coefficients obey an energy conservation condition:

$$
r_{i}^{2}+t_{i}^{2}=1
$$

The transmission transfer function denominator polynomial for one stage is

$$
1+r_{0} r_{1} z^{-1}
$$

for two stages is

$$
1+\left(r_{0} r_{1}+r_{1} r_{2}\right) z^{-1}+r_{0} r_{2} z^{-2}
$$

and for three stages is

$1+\left(r_{0} r_{1}+r_{1} r_{2}+r_{2} r_{3}\right) z^{-1}+\left(r_{0} r_{2}+r_{0} r_{1} r_{2} r_{3}+r_{1} r_{3}\right) z^{-2}+r_{0} r_{3} z^{-3}$.

Simple induction can derive the polynomial for $n$ stages.

In this paper we include gain as well as delay between the interfaces, and this block diagram for an active lattice filter is shown in Figure 3. Equations (2), (3), and (4) may be readily generalized to include this gain. For example, the transmission transfer function denominator polynomial for one stage with gains is

$$
1+r_{0} r_{1} G_{1}^{2} z^{-1}
$$

for two stages with gains is

$$
1+\left(r_{0} r_{1} G_{1}^{2}+r_{1} r_{2} G_{2}^{2}\right) z^{-1}+r_{0} r_{2} G_{1}^{2} G_{2}^{2} z^{-2},
$$

and for three stages with gains is

$$
\begin{aligned}
1+ & \left(r_{0} r_{1} G_{1}^{2}+r_{1} r_{2} G_{2}^{2}+r_{2} r_{3} G_{3}^{2}\right) z^{-1} \\
& +\left(r_{0} r_{2} G_{1}^{2} G_{2}^{2}+r_{0} r_{1} r_{2} r_{3} G_{1}^{2} G_{3}^{2}+r_{1} r_{3} G_{2}^{2} G_{3}^{2}\right) z^{-2} \\
& +r_{0} r_{3} G_{1}^{2} G_{2}^{2} G_{3}^{2} z^{-3} .
\end{aligned}
$$

Again, induction can be used to derive the polynomial for $n$ stages. The gain for every stage appears to a power 2 , regardless of the number of stages used.

Given a desired polynomial of degree $n$ we can use the layer-peeling process $[1,2,3]$ to compute $r_{0}, r_{1}, \ldots, r_{n}$ (actually $r_{0}$ can be chosen and the other reflection coefficients are computed). Fixing these reflection coefficients we can simply write the one-stage polynomial with gains as

$$
1+c_{1} G_{1}^{2} z^{-1}
$$




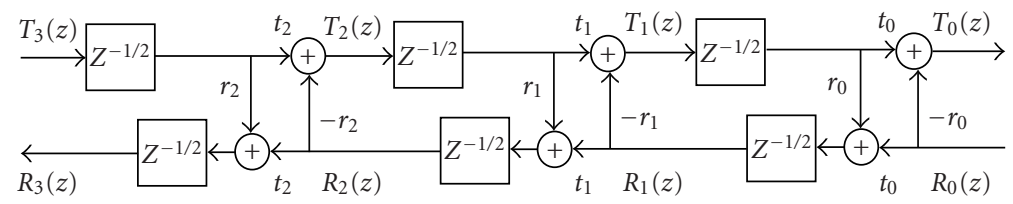

FIgURE 2: Signal flow diagram for three stages of an optical lattice filter.

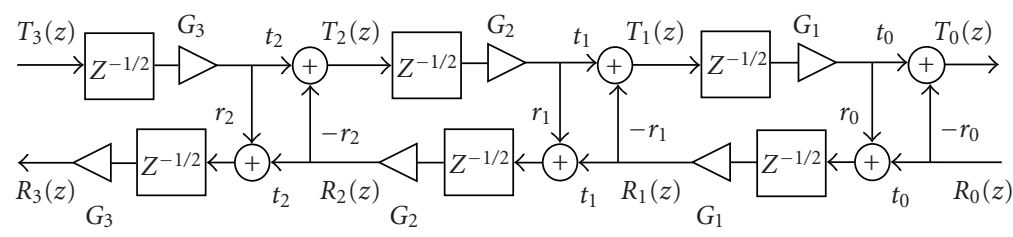

FIGURE 3: Signal flow diagram for three stages of an active optical lattice filter.

the two-stage polynomial with gains as

$$
1+\left(c_{1} G_{1}^{2}+c_{2} G_{2}^{2}\right) z^{-1}+c_{12} G_{1}^{2} G_{2}^{2} z^{-2},
$$

and the three-stage polynomial with gains as

$$
\begin{aligned}
1 & +\left(c_{1} G_{1}^{2}+c_{2} G_{2}^{2}+c_{3} G_{3}^{2}\right) z^{-1} \\
& +\left(c_{12} G_{1}^{2} G_{2}^{2}+c_{13} G_{1}^{2} G_{3}^{2}+c_{23} G_{2}^{2} G_{3}^{2}\right) z^{-2}+c_{123} G_{1}^{2} G_{2}^{2} G_{3}^{2} z^{-3} .
\end{aligned}
$$

Here the c's are computed from the r's in the obvious way. The $n$ th-degree polynomial with gains is

$$
\begin{aligned}
1 & +\left(\sum_{i=1}^{n} c_{i} G_{i}^{2}\right) z^{-1}+\left(\sum_{i, j=1, i<j}^{n} c_{i j} G_{i}^{2} G_{j}^{2}\right) z^{-2} \\
& +\cdots+c_{12 \cdots n} G_{1}^{2} G_{2}^{2} \cdots G_{n}^{2} z^{-n} .
\end{aligned}
$$

Again the $c$ coefficients are known numbers computed from the $r$ 's, and the gains $G_{i}$ are allowed to vary. Since we are interested in only nonnegative real gains $G_{i}$ we set $x_{i}=G_{i}^{2}$ and replace (11) by

$$
\begin{aligned}
1 & +\left(\sum_{i=1}^{n} c_{i} x_{i}\right) z^{-1}+\left(\sum_{i, j=1, i<j}^{n} c_{i j} x_{i} x_{j}\right) z^{-2} \\
& +\cdots+c_{12 \cdots n} x_{1} x_{2} \cdots x_{n} z^{-n} .
\end{aligned}
$$

If we determine $x_{1}, x_{2}, \ldots, x_{n}$ then we know $G_{1}, G_{2}, \ldots, G_{n}$. We first consider a two-stage filter and return to the general case later.

\section{TWO-STAGE EXAMPLE}

In the case of a two-stage active lattice filter, (12) becomes

$$
1+\left(c_{1} x_{1}+c_{2} x_{1}\right) z^{-1}+c_{12} x_{1} x_{2} z^{-2}
$$

where the c's are fixed and only the $x_{i}$ can vary. If a desired denominator polynomial is

$$
1+a_{1} z^{-1}+a_{2} z^{-2}
$$

then the equations to determine the gains as function of the polynomial coefficients are

$$
\begin{aligned}
c_{1} x_{1}+c_{2} x_{1} & =a_{1}, \\
c_{12} x_{1} x_{2} & =a_{2} .
\end{aligned}
$$

Geometrically, these equations represent a straight line and a hyperbola in the closed first quadrant in $\left(x_{1}, x_{2}\right)$ space. With $c_{1}, c_{2}, c_{12}$ determined and $a_{1}, a_{2}$ given, a solution is a point $\left(x_{1}, x_{2}\right)$ where the line and the hyperbola intersect. These equations can also be thought of as a map from the gains (actually gains squared) space $\left(x_{1}, x_{2}\right)$ to the desired polynomial coefficient space $\left(a_{1}, a_{2}\right)$.

For example, if we set $r_{0}=1, r_{1}=1 / 4$, and $r_{2}=1 / 4$, (3) for the transfer function denominator becomes

$$
1+\left(\frac{5}{16}\right) z^{-1}+\left(\frac{1}{4}\right) z^{-2}
$$

This discussion could have begun from the point of view of choosing coefficients $a_{1}=5 / 16, a_{2}=1 / 4$, and $r_{0}=1$, and using the Dowling-MacFarlane layer-peeling algorithm to find $r_{1}=1 / 4$ and $r_{2}=1 / 4$. We have stable systems in transmission and reflection since both poles are inside the unit circle. Equations (15) now becomes

$$
\begin{gathered}
\left(\frac{1}{4}\right) x_{1}+\left(\frac{1}{16}\right) x_{2}=a_{1}, \\
\left(\frac{1}{4}\right) x_{1} x_{2}=a_{2} .
\end{gathered}
$$

With $a_{1}=5 / 16$ and $a_{2}=1 / 4$ we have two solutions $(1,1)$ and $(1 / 4,4)$ for $\left(x_{1}, x_{2}\right)$. The solution of interest to us 


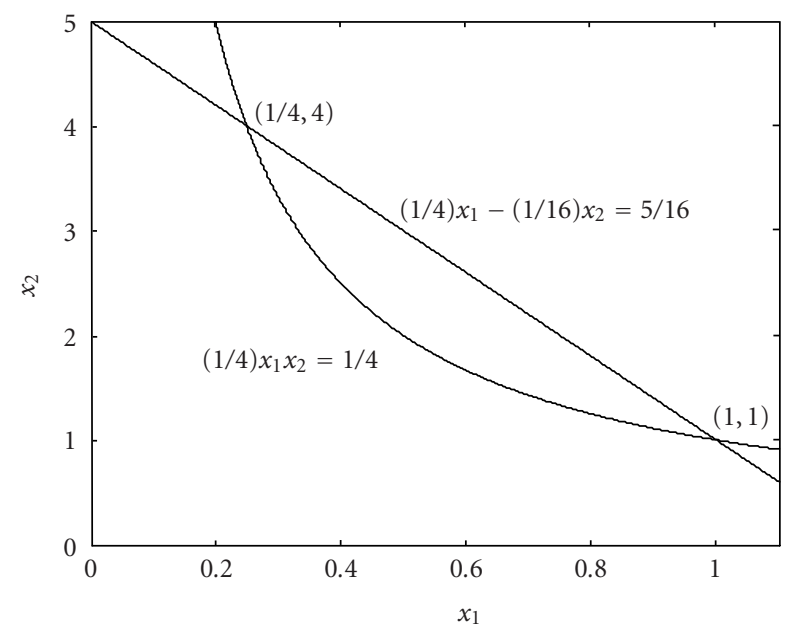

FIGURE 4: Intersection in $\left(x_{1}, x_{2}\right)$ space for $a_{1}=5 / 16$ and $a_{2}=1 / 4$.

is $(1,1)$. Considering $(17)$ as a map from $\left(x_{1}, x_{2}\right)$ space to $\left(a_{1}, a_{2}\right)$ space, the Jacobian matrix is $\left[\begin{array}{cc}1 / 4 & 1 / 16 \\ (1 / 4) x_{2} & (1 / 4) x_{1}\end{array}\right]$. We note that this matrix, evaluated at $\left(x_{1}, x_{2}\right)=(1,1)$, is nonsingular. Thus by the inverse function theorem [9] there is an open set $U$ containing $(1,1)$ in $\left(x_{1}, x_{2}\right)$ space and open set $V$ containing $(5 / 16,1 / 4)$ in $\left(a_{1}, a_{2}\right)$ space. Hence for each coefficient pair $\left(a_{1}, a_{2}\right)$ in $V$ there is exactly one set of gains (squared) $\left(x_{1}, x_{2}\right)$ in $U$ that solves (17). Geometrically, for $a_{1}=5 / 16$ and $a_{2}=1 / 4$ the line and the hyperbola intersect transversally (nontangentially) at $(1,1)$ in $\left(x_{1}, x_{2}\right)$ space. If we change $a_{1}$ and $a_{2}$ slightly we still have a transverse intersection near $(1,1)$. This intersection is shown graphically in Figure 4.

We now show that it is not possible to find the gains in (17) to do arbitrary pole placement for the same values of $r_{0}$, $r_{1}$, and $r_{2}$. If we let $a_{1}=5 / 16$ and $a_{2}=1$ in (17), then

$$
\begin{gathered}
\left(\frac{1}{4}\right) x_{1}+\left(\frac{1}{16}\right) x_{2}=\frac{5}{16} \\
\left(\frac{1}{4}\right) x_{1} x_{2}=1
\end{gathered}
$$

have only complex solutions for $x_{1}$ and $x_{2}$. Of course the straight line and hyperbola in (17) do not intersect in $\left(x_{1}, x_{2}\right)$ space. Hence arbitrary denominator polynomials cannot be realized for the transmission and reflection transfer functions if the reflection coefficients are held fixed and the gains varied, for given order (number of lattice stages).
There is one more case of interest. If $a_{1}=1 / 2$ and $a_{2}=$ $1 / 4$ in (17), then

$$
\begin{gathered}
\left(\frac{1}{4}\right) x_{1}+\left(\frac{1}{16}\right) x_{2}=\frac{1}{2}, \\
\left(\frac{1}{4}\right) x_{1} x_{2}=1
\end{gathered}
$$

have only the solution $x_{1}=1$ and $x_{2}=4$. Hence the straight line and the hyperbola from (17) intersect at only one point, a point of tangency. Slight changes in $a_{1}$ from $1 / 2$ can result in either no points of intersection or two points of intersection. It is very important to note that the Jacobian matrix $\left[\begin{array}{cc}1 / 4 & 1 / 16 \\ (1 / 4) x_{2} & (1 / 4) x_{1}\end{array}\right]$ of $(17)$ is singular at the point $\left(x_{1}, x_{2}\right)=(1,4)$.

\section{MAIN RESULTS}

We return to the general case for $n$ stages with gains in Section 3. Equation (12) of interest is

$$
\begin{aligned}
1 & +\left(\sum_{i=1}^{n} c_{i} x_{i}\right) z^{-1}+\left(\sum_{i, j=1, i<j}^{n} c_{i j} x_{i} x_{j}\right) z^{-2} \\
& +\cdots+c_{12 \ldots n} x_{1} x_{2} \cdots x_{n} z^{-n} .
\end{aligned}
$$

Here the c's (through the $r$ 's) have been chosen by using layer peeling to deliver a specified transmission transfer function denominator without using the gains (or equivalently, when all the $x_{i}=1$ ). If another desired denominator polynomial to be achieved by computing only the gains is

$$
1+a_{1} z^{-1}+a_{2} z^{-2}+\cdots+a_{n} z^{-n},
$$

then the equations to determine the gains as functions of the polynomial coefficients are

$$
\begin{gathered}
\left(\sum_{i=1}^{n} c_{i} x_{i}\right)=a_{1}, \\
\left(\sum_{i, j=1, i<j}^{n} c_{i j} x_{i} x_{j}\right)=a_{2}, \\
\vdots \\
c_{12 \cdots n} x_{1} x_{2} \cdots x_{n}=a_{n} .
\end{gathered}
$$

The $n$ by $n$ Jacobian matrix of the gains (or gains squared) to polynomial coefficient map is

$$
\mathbf{J}=\left[\begin{array}{cccc}
c_{1} & c_{2} & \cdots & c_{n} \\
\sum_{j=2}^{n} c_{1 j} x_{j} & c_{12} x_{1}+\sum_{j=3}^{n} c_{2 j} x_{j} & \cdots & \sum_{i=1}^{n-1} c_{i n} x_{i} \\
\vdots & \vdots & \ddots & \vdots \\
c_{12 \cdots n} x_{2} x_{3} \cdots x_{n} & c_{12 \cdots n} x_{1} x_{3} \cdots x_{n} & \cdots & c_{12 \cdots n} x_{1} x_{2} \cdots x_{n-1}
\end{array}\right]
$$


which is easily computed by taking partial derivatives in (22). When all gains (squared) $x_{i}$ in (22) are unity, we denote the corresponding polynomial coefficients by $\hat{a}_{1}, \hat{a}_{2}, \ldots, \hat{a}_{n}$. By the inverse function theorem [9], these are the coefficients of the transfer function denominator polynomial for which the reflection coefficients are fixed.

If we move the $a_{i}$ from the right-hand side to the lefthand side in (22), we have a system of equations of the form

$$
\begin{gathered}
f_{1}\left(x_{1}, x_{2}, \ldots, x_{n}\right)=0, \\
f_{2}\left(x_{1}, x_{2}, \ldots, x_{n}\right)=0, \\
\vdots \\
f_{n}\left(x_{1}, x_{2}, \ldots, x_{n}\right)=0 .
\end{gathered}
$$

Equations (24) may be solved by the Newton-Raphson algorithm to find the $\left(x_{1}, x_{2}, \ldots, x_{n}\right)$ as a function of the $\left(a_{1}, a_{2}, \ldots, a_{n}\right)$, so long as the Jacobian matrix of this system is nonsingular.

As an example, we now return to the two-stage example in Section 4 and apply the Newton-Raphson algorithm to compute the correct gains as we vary the desired polynomial coefficients. We use Matlab to implement Newton-Raphson [13]. Equations (17) of interest are

$$
\begin{gathered}
\left(\frac{1}{4}\right) x_{1}+\left(\frac{1}{16}\right) x_{2}=a_{1}, \\
\left(\frac{1}{4}\right) x_{1} x_{2}=a_{2} .
\end{gathered}
$$

The starting values are $a_{1}=5 / 16$ and $a_{2}=1 / 4$ for which the solutions are $x_{1}=1$ and $x_{2}=1$. Of course this implies that $G_{1}=1$ and $G_{2}=1$. To make the changes in $a_{1}$ and $a_{2}$ somewhat random we choose

$$
\begin{aligned}
& a_{1}=\frac{5}{16} \pm 0.05 \times(\text { rand }-0.5), \\
& a_{2}=\frac{1}{4} \pm 0.05 \times(\text { rand }-0.5),
\end{aligned}
$$

where rand is a Matlab command for a uniformly distributed random variable with values between 0 and 1 . Then we solve for $x_{1}, x_{2}, G_{1}$, and $G_{2}$. Our computations yield the results in Table 1.

The Newton-Raphson algorithm actually provides two solutions $\left(x_{1}, x_{2}\right)$ for each $a_{1}, a_{2}$, but we take only that solution which is on the same side of the straight line $x_{2}=4 x_{1}$ as the point $(1,1)$ (recall that we work in an open neighborhood of $(1,1))$. The line $x_{2}=4 x_{1}$ represents the set of points where the Jacobin matrix $\left[\begin{array}{cc}1 / 4 & 1 / 16 \\ (1 / 4) x_{2} & (1 / 4) x_{1}\end{array}\right]$ is singular.

These results follow from the point of view that given the desired second-degree denominator polynomial, we program the gains to achieve that polynomial. We next merge
TABLE 1: Filter coefficients and gains.

\begin{tabular}{cccccc}
\hline$a_{1}$ & $a_{2}$ & $x_{1}$ & $x_{2}$ & $G_{1}$ & $G_{2}$ \\
\hline $5 / 16$ & $1 / 4$ & 1 & 1 & 1 & 1 \\
0.3350 & 0.2366 & 1.13 & 0.83 & 1.063 & 0.910 \\
0.31780 & 0.2493 & 1.028 & 0.969 & 1.013 & 0.984 \\
0.3321 & 0.2631 & 1.086 & 0.968 & 1.042 & 0.983 \\
0.3103 & 0.2259 & 1.019 & 0.886 & 1.009 & 0.9412 \\
0.3286 & 0.2472 & 1.08 & 0.909 & 1.039 & 0.953 \\
0.3067 & 0.2646 & 0.947 & 1.116 & 0.973 & 1.056 \\
0.2914 & 0.2619 & 0.861 & 1.215 & 0.9279 & 1.1022 \\
0.3172 & 0.2338 & 1.045 & 0.894 & 1.022 & 0.945 \\
0.2907 & 0.2708 & 0.840 & 1.28 & 0.916 & 1.131 \\
0.3170 & 0.2697 & 0.997 & 1.081 & 0.998 & 1.039 \\
0.3346 & 0.2574 & 1.10 & 0.931 & 1.048 & 0.964 \\
0.2968 & 0.2245 & 0.872 & 1.25 & 0.933 & 1.118 \\
0.3306 & 0.2649 & 1.076 & 0.984 & 1.037 & 0.991 \\
0.3276 & 0.2448 & 1.084 & 0.902 & 1.041 & 0.949 \\
0.3239 & 0.2651 & 1.04 & 1.018 & 1.019 & 1.008 \\
0.3152 & 0.2623 & 0.998 & 1.051 & 0.998 & 1.025 \\
0.2909 & 0.2483 & 0.882 & 1.125 & 0.939 & 1.06 \\
0.3166 & 0.2673 & 0.998 & 1.07 & 0.998 & 1.034 \\
0.3112 & 0.2351 & 1.01 & 0.928 & 1.004 & 0.963 \\
\hline
\end{tabular}

our gain computation procedure with an adaptive process so that we start with data, identify the AR transfer function, and compute the appropriate gains.

We generate data according to the linear time-varying difference equation

$$
\begin{aligned}
& y(n)+\left(\frac{13}{24}\right) y(n-1)+\left(\frac{5}{8}\right) y(n-2) \\
& +\left[\frac{8}{27}+\frac{1}{27} \cos (n-3)\right] y(n-3)=u(n)
\end{aligned}
$$

with zero initial conditions $y(-1)=y(-2)=y(-3)=0$ and input $u(n)=\delta(n)$, the discrete delta function. We assume that the data is associated with the time-invariant linear system

$$
y(n)+a_{1} y(n-1)+a_{2} y(n-2)+a_{3} y(n-3)=u(n)
$$

and we identify $a_{1}, a_{2}, a_{3}$ from the data as $n$ moves. These $a_{1}, a_{2}, a_{3}$ are the coefficients of the desired AR denominator polynomial $1+a_{1} z^{-1}+a_{2} z^{-2}+a_{3} z^{-3}$. Then we compute the $x_{1}, x_{2}, x_{3}$ (and hence the gains $G_{1}, G_{2}, G_{3}$ ) from the equations

$$
\begin{gathered}
c_{1} x_{1}+c_{2} x_{2}+c_{3} x_{3}=a_{1}, \\
c_{12} x_{1} x_{2}+c_{13} x_{1} x_{3}+c_{23} x_{2} x_{3}=a_{2}, \\
c_{123} x_{1} x_{2} x_{3}=a_{3} .
\end{gathered}
$$


For the starting point we take the difference equation

$$
\begin{aligned}
y(n) & +\left(\frac{13}{24}\right) y(n-1)+\left(\frac{5}{8}\right) y(n-2)+\left(\frac{1}{3}\right) y(n-3) \\
= & u(n)
\end{aligned}
$$

and thus the polynomial $1+(13 / 24) z^{-1}+(5 / 8) z^{-2}+(1 / 3) z^{-3}$ results. This difference equation can be found by substituting $u(n)=\delta(n)$ into (27) with zero initial conditions, evaluating $y(0), y(1), y(2), y(3)$, and computing $a_{1}, a_{2}, a_{3}$ from (28) using 3 linear equations (for $y(n)=y(1), y(2), y(3)$ ) in 3 unknowns. Choosing $r_{0}=1$ we compute the reflection coef- ficients $r_{1}=1 / 4, r_{2}=1 / 2, r_{3}=1 / 3$ by layer peeling. Thus (29) becomes (see (7) and (10))

$$
\begin{gathered}
\left(\frac{1}{4}\right) x_{1}+\left(\frac{1}{8}\right) x_{2}+\left(\frac{1}{6}\right) x_{3}=a_{1} \\
\left(\frac{1}{2}\right) x_{1} x_{2}+\left(\frac{1}{24}\right) x_{1} x_{3}+\left(\frac{1}{12}\right) x_{2} x_{3}=a_{2} \\
\left(\frac{1}{3}\right) x_{1} x_{2} x_{3}=a_{3}
\end{gathered}
$$

The Jacobian matrix is

$$
\left[\begin{array}{lll}
\left(\frac{1}{4}\right) & \left(\frac{1}{8}\right) & \left(\frac{1}{6}\right) \\
\left(\frac{1}{2}\right) x_{2}+\left(\frac{1}{24}\right) x_{3} & \left(\frac{1}{2}\right) x_{1}+\left(\frac{1}{12}\right) x_{3} & \left(\frac{1}{24}\right) x_{1}+\left(\frac{1}{12}\right) x_{2} \\
\left(\frac{1}{3}\right) x_{2} x_{3} & \left(\frac{1}{3}\right) x_{1} x_{3} & \left(\frac{1}{3}\right) x_{1} x_{2}
\end{array}\right] .
$$

As discussed above, this matrix is nonsingular when $x_{1}, x_{2}$, $x_{3}$ are all unity. We then substitute in the varying values for $a_{1}, a_{2}, a_{3}$ and compute the corresponding $x_{1}, x_{2}, x_{3}$ (from (31)) and $G_{1}, G_{2}, G_{3}$. The first computation is for $a_{1}=13 / 24$, $a_{2}=5 / 8$, and $a_{3}=1 / 3$, and the solution is $x_{1}=1, x_{2}=1$, and $x_{3}=1$.

The second computation involves $a_{1}, a_{2}, a_{3}$ calculated by substituting $u(n)=\delta(n)$ into (27) with zero initial conditions, evaluating $y(0), y(1), y(2), y(3), y(4)$, and computing $a_{1}, a_{2}, a_{3}$ from (28) using 3 linear equations (for $y(n)=$ $y(2), y(3), y(4))$ in 3 unknowns. The third and higher computations proceed in the obvious way. We record our results in Table 2

For this example we could have driven the system by noise, computed the autocorrelations, arrived at the polynomial coefficients through standard algorithms, and then computed the gains using our technique. Moreover, we also could have estimated the polynomial coefficients using a recursive least squares method based on the Kalman filter. However, we decided to keep things simple and work straight with the data from the difference equation, using sets of 3 equations in 3 unknowns. With noisy data we also could have used more linear equations in the 3 unknowns and found pseudoinverse solutions.

\section{PARAMETER SENSITIVITY ANALYSIS}

The goal is to quantify the variation of denominator coefficients as the gains vary. Since the gains are physically realized their numerical value is prone to change. This might result in a significantly different transfer function. Hence the perturbation of the coefficients $a_{i}$ 's due to error in the realization of the gains $G_{j}$ 's is worth analyzing. We quantify the perturbation relations by a measurable parameter called sensitivity. For ease of notation we analyze the sensitivity of the coefficients $a_{i}$ with respect to the gains squared $x_{j}$.

The traditional definition of sensitivity of a parameter $a$ with respect to a parameter $x$ is given by

$$
S=\frac{d a / a}{d x / x}
$$

This can be viewed as the relative change of $a$ with respect to change in $x$. Low values of $S$ means the coefficients are somewhat insensitive to gain changes. The next step is to see how $S$ fits into the nonlinear gains squared-coefficients relations.

The general gain squared-coefficients relation according to $(22)$ can be expressed in the form

$$
\begin{gathered}
f_{1}\left(x_{1}, x_{2}, \ldots, x_{n}\right)=a_{1}, \\
f_{2}\left(x_{1}, x_{2}, \ldots, x_{n}\right)=a_{2}, \\
\vdots \\
f_{n}\left(x_{1}, x_{2}, \ldots, x_{n}\right)=a_{n} .
\end{gathered}
$$

The above set of equations can be represented in a vector form as

$$
f(\mathbf{x})=\mathbf{a},
$$

where $\mathbf{x}=\left[x_{1}, \ldots, x_{n}\right]^{T}$ and $\mathbf{a}=\left[a_{1}, \ldots, a_{n}\right]^{T}$ and $T$ is the matrix-transpose operator. 
TABLE 2: Filter coefficients and gains.

\begin{tabular}{|c|c|c|c|c|c|c|c|c|}
\hline$a_{1}$ & $a_{2}$ & $a_{3}$ & $x_{1}$ & $x_{2}$ & $x_{3}$ & $G_{1}$ & $G_{2}$ & $G_{3}$ \\
\hline $13 / 24$ & $5 / 8$ & $1 / 3$ & 1 & 1 & 1 & 1 & 1 & 1 \\
\hline $13 / 24$ & $5 / 8$ & 0.3163 & 1.06948 & 0.95293 & 0.93108 & 1.0342 & 0.9762 & 0.9649 \\
\hline $13 / 24$ & $5 / 8$ & 0.2809 & 1.19 & 0.883 & 0.8006 & 1.0909 & 0.9397 & 0.8948 \\
\hline $13 / 24$ & $5 / 8$ & 0.2596 & 1.255 & 0.852 & 0.7275 & 1.1203 & 0.9230 & 0.8529 \\
\hline $13 / 24$ & $5 / 8$ & 0.2721 & 1.2182 & 0.8702 & 0.7699 & 1.1037 & 0.9328 & 0.8774 \\
\hline $13 / 24$ & $5 / 8$ & 0.3068 & 1.1045 & 0.93142 & 0.89466 & 1.0509 & 0.9651 & 0.9459 \\
\hline $13 / 24$ & $5 / 8$ & 0.3319 & 1.00627 & 0.9954 & 0.9938 & 1.0031 & 0.9977 & 0.9969 \\
\hline $13 / 24$ & $5 / 8$ & 0.3242 & 1.03851 & 0.9731 & 0.96238 & 1.0191 & 0.9865 & 0.981 \\
\hline $13 / 24$ & $5 / 8$ & 0.2909 & 1.1589 & 0.90059 & 0.83612 & 1.0765 & 0.94899 & 0.91439 \\
\hline $13 / 24$ & $5 / 8$ & 0.2626 & 1.24651 & 0.85683 & 0.73761 & 1.1130 & 0.9276 & 0.8639 \\
\hline $13 / 24$ & $5 / 8$ & 0.2652 & 1.23887 & 0.86039 & 0.74641 & 1.0678 & 0.9544 & 0.9254 \\
\hline $13 / 24$ & $5 / 8$ & 0.2965 & 1.0 .1487 & 0.98940 & 0.98565 & 1.0074 & 0.9946 & 0.9928 \\
\hline $13 / 24$ & $5 / 8$ & 0.3299 & 1.12353 & 0.92031 & 0.87447 & 1.0599 & 0.9607 & 0.93513 \\
\hline $13 / 24$ & $5 / 8$ & 0.3014 & 1.22996 & 0.8646 & 0.75661 & 1.1090 & 0.9298 & 0.8698 \\
\hline $13 / 24$ & $5 / 8$ & 0.2682 & 1.25177 & 0.85441 & 0.73155 & 1.1188 & 0.9243 & 0.8553 \\
\hline $13 / 24$ & $5 / 8$ & 0.2608 & 1.17455 & 0.89230 & 0.81895 & 1.0838 & 0.9446 & 0.9049 \\
\hline $13 / 24$ & $5 / 8$ & 0.2861 & 1.05207 & 0.96415 & 0.94878 & 1.0257 & 0.9819 & 0.9924 \\
\hline $13 / 24$ & $5 / 8$ & 0.3329 & 1.08782 & 0.94150 & 0.91214 & 1.0429 & 0.9703 & 0.955 \\
\hline $13 / 24$ & $5 / 8$ & 0.3114 & 1.20631 & 0.8761 & 0.78347 & 1.0983 & 0.9360 & 0.8851 \\
\hline $13 / 24$ & $5 / 8$ & 0.2760 & 1.25612 & 0.85242 & 0.72651 & 1.1207 & 0.9233 & 0.8523 \\
\hline
\end{tabular}

Writing (35) in differential form we get

$$
\mathbf{J d}_{\mathbf{x}}=\mathbf{d}_{\mathrm{a}}
$$

where $\mathbf{d}_{\mathbf{x}}=\left[d x_{1}, \ldots, d x_{n}\right]^{T}, \mathbf{d}_{\mathbf{a}}=\left[d a_{1}, \ldots, d a_{n}\right]^{T}$, and

$$
\mathbf{J}=\left[\begin{array}{cccc}
\frac{\partial f_{1}(\mathbf{x})}{\partial x_{1}} & \frac{\partial f_{1}(\mathbf{x})}{\partial x_{2}} & \cdots & \frac{\partial f_{1}(\mathbf{x})}{\partial x_{n}} \\
\frac{\partial f_{2}(\mathbf{x})}{\partial x_{1}} & \frac{\partial f_{2}(\mathbf{x})}{\partial x_{2}} & \cdots & \frac{\partial f_{2}(\mathbf{x})}{\partial x_{n}} \\
\cdot & \cdot & \cdot & \cdot \\
\cdot & \cdot & \cdot & \cdot \\
\cdot & \cdot & \cdot & \cdot \\
\frac{\partial f_{n}(\mathbf{x})}{\partial x_{1}} & \frac{\partial f_{n}(\mathbf{x})}{\partial x_{2}} & \cdots & \frac{\partial f_{n}(\mathbf{x})}{\partial x_{n}}
\end{array}\right],
$$

where $\mathbf{J}$ is the Jacobian matrix. The entries of the matrix $\mathbf{J}$ are $\mathbf{J}_{i, j}$. It is easy to see from (36) that if only $x_{j}$ changes, then

$$
\mathbf{J}_{i, j} \mathbf{d}_{\mathbf{x}_{j}}=\mathbf{d}_{\mathbf{a}_{i}}
$$

Plugging (38) into (33) and a little manipulation gives

$$
\mathbf{S}_{i, j}=\frac{\mathbf{J}_{i, j} \mathbf{x}_{j}}{\mathbf{a}_{i}}
$$

where $\boldsymbol{S}_{i, j}$ relates the sensitivity of $\mathbf{a}_{i}$ to changes in $\mathbf{x}_{j}$. Thus we can find a sensitivity matrix $\mathbf{S}$ with $\boldsymbol{S}_{i, j}$ as its elements. $\mathbf{S}$ gives the one-to-one sensitivity relations between the square of the gains and the coefficients.

An upper bound on the sensitivity matrix, when one or more gains vary, is estimated using the 2-norm as follows. Equation (36) can be written as

$$
\mathbf{J d}_{\mathbf{x}}=\mathbf{d}_{\mathrm{a}}
$$

Applying norm to both sides of the equation we get

$$
\left\|\mathbf{J d}_{\mathbf{x}}\right\|=\left\|\mathbf{d}_{\mathbf{a}}\right\| \Longrightarrow\left\|\mathbf{d}_{\mathbf{a}}\right\| \leq\|\mathbf{J}\|\left\|\mathbf{d}_{\mathbf{x}}\right\|
$$

Here $\|\cdot\|$ denotes $\sqrt{\max \left(\text { eigenvalues of } \mathbf{J}^{T} \mathbf{J}\right)}$ for matrices and 2-norm for vectors. Thus we get

$$
\frac{\left\|\mathbf{d}_{\mathbf{a}}\right\|}{\left\|\mathbf{d}_{\mathbf{x}}\right\|} \leq\|\mathbf{J}\|
$$

or equivalently,

$$
\frac{\left\|\mathbf{d}_{\mathbf{a}}\right\| /\|\mathbf{a}\|}{\left\|\mathbf{d}_{\mathbf{x}}\right\| /\|\mathbf{x}\|}=s \leq \frac{\|\mathbf{J}\|\|\mathbf{x}\|}{\|\mathbf{a}\|}
$$

Here $s$ is sensitivity with respect to gain squared. Thus (43) gives an upper bound on sensitivity. The sensitivity bound $(s)$ is tabulated in Table 3 for different gains squared. 
TABLE 3: Sensitivity of filter parameters respective to gains.

\begin{tabular}{ccccccc}
\hline$a_{1}$ & $a_{2}$ & $a_{3}$ & $x_{1}$ & $x_{2}$ & $x_{3}$ & $s$ \\
\hline $13 / 24$ & $5 / 8$ & $1 / 3$ & 1 & 1 & 1 & 1.9702 \\
$13 / 24$ & $5 / 8$ & 0.3163 & 1.06948 & 0.95293 & 0.93108 & 1.9359 \\
$13 / 24$ & $5 / 8$ & 0.2809 & 1.19 & 0.883 & 0.8006 & 1.9109 \\
$13 / 24$ & $5 / 8$ & 0.2596 & 1.255 & 0.852 & 0.7275 & 1.9139 \\
$13 / 24$ & $5 / 8$ & 0.2721 & 1.2182 & 0.8702 & 0.7699 & 1.9109 \\
$13 / 24$ & $5 / 8$ & 0.3068 & 1.1045 & 0.93142 & 0.89466 & 1.9243 \\
$13 / 24$ & $5 / 8$ & 0.3319 & 1.00627 & 0.9954 & 0.9938 & 1.9664 \\
$13 / 24$ & $5 / 8$ & 0.3242 & 1.03851 & 0.9731 & 0.96238 & 1.9492 \\
$13 / 24$ & $5 / 8$ & 0.2909 & 1.1589 & 0.90059 & 0.83612 & 1.9135 \\
$13 / 24$ & $5 / 8$ & 0.2626 & 1.24651 & 0.85683 & 0.73761 & 1.9129 \\
$13 / 24$ & $5 / 8$ & 0.2652 & 1.23887 & 0.86039 & 0.74641 & 1.9121 \\
$13 / 24$ & $5 / 8$ & 0.2965 & 1.0 .1487 & 0.9894 & 0.98565 & 1.9162 \\
$13 / 24$ & $5 / 8$ & 0.3299 & 1.12353 & 0.92031 & 0.87447 & 1.9615 \\
$13 / 24$ & $5 / 8$ & 0.3014 & 1.22996 & 0.8646 & 0.75661 & 1.9196 \\
$13 / 24$ & $5 / 8$ & 0.2682 & 1.25177 & 0.85441 & 0.73155 & 1.9115 \\
$13 / 24$ & $5 / 8$ & 0.2608 & 1.17455 & 0.8923 & 0.81895 & 1.9135 \\
$13 / 24$ & $5 / 8$ & 0.2861 & 1.05207 & 0.96415 & 0.94878 & 1.9119 \\
$13 / 24$ & $5 / 8$ & 0.3329 & 1.08782 & 0.9415 & 0.91214 & 1.969 \\
$13 / 24$ & $5 / 8$ & 0.3114 & 1.20631 & 0.8761 & 0.78347 & 1.9294 \\
$13 / 24$ & $5 / 8$ & 0.276 & 1.25612 & 0.85242 & 0.72651 & 1.9106 \\
\hline & & & & & &
\end{tabular}

The results in Table 3 show that the active lattice is reasonably robust with respect to gain tolerances. That is to say that the filter response does not appreciably change with small injection current uncertainties. This advantageous result is somewhat expected given the traditional lattice filter's robustness with respect to round off error or the reflection coefficient variations $[2,4,13]$.

\section{CONCLUSIONS AND FUTURE RESEARCH}

We have added gains to the optical lattice filters $[1,2,3]$ in order to make the response functions programmable. To determine the denominator polynomials achievable by gains only we set up a map from the gains (squared) to the polynomial coefficients. Applying the inverse function theorem we provide a result giving sufficient conditions that all polynomials in an open set can be generated by appropriated choices of gains. This result naturally leads to a method for computing the gains using the Newton-Raphson algorithm. Two interesting examples are given, one of which stressed the adaptive signal processing point of view. We also performed a sensitivity analysis, measuring how the denominator coefficients vary as the gains vary.

We have shown that the presence of gains in the lattice filter can provide additional flexibility in the filter response. These gains may be implemented in a semiconductor laser amplifier, and hence these will be adjustable at $\mathrm{GHz}$ rates. In accompanying experimental work, we will be building and testing these devices, and exploring their range of operation both theoretically and empirically. This range will be bounded on one end by the noise figure and stability on the other. In addition, theoretical and experimental work is also underway in two-dimensional active lattice filters based on four-directional couplers [14].

\section{REFERENCES}

[1] D. L. MacFarlane and E. M. Dowling, "Z-domain techniques in the analysis of Fabry-Perot etalons and multilayer structures," Journal of the Optical Society of America $\{A\}$, vol. 11, no. 1, pp. 236-245, 1994.

[2] E. M. Dowling and D. L. MacFarlane, "Lightwave lattice filters for optically multiplexed communication systems," J. Lightwave Technol., vol. 12, no. 3, pp. 471-486, 1994.

[3] D. L. MacFarlane, E. M. Dowling, and V. Narayan, "Ring resonators with $N \times M$ couplers," Fiber and Integrated Optics, vol. 14, no. 3, pp. 195-210, 1995.

[4] S. Haykin, Adaptive Filter Theory, Prentice Hall, Englewood Cliffs, NJ, USA, 4th edition, 2002.

[5] P. A. Regalia, Adaptive IIR Filtering in Signal Processing and Control, Marcel Dekker, New York, NY, USA, 1995.

[6] M. J. Maron and R. J. Lopez, Numerical Analysis: A Practical Approach, Wadsworth, Belmont, Calif, USA, 3rd edition, 1991.

[7] J. E. Dennis Jr. and R. B. Schnabel, Numerical Methods for Unconstrained Optimization and Nonlinear Equations, Prentice Hall, Englewood Cliffs, NJ, USA, 1983.

[8] G. Evans, N. W. Carlson, J. M. Hammer, and J. K. Butler, "Grating surface emitting lasers," in Chapter 4 of Surface Emitting Semiconductor Lasers and Arrays, G. A. Evans and J. M. Hammer, Eds., Academic Press, New York, NY, USA, 1993.

[9] W. Rudin, Principles of Mathematical Analysis, McGraw-Hill, New York, NY, USA, 2nd edition, 1964.

[10] B. Moslehi, J. W. Goodman, M. Tur, and H. J. Shaw, "Fiberoptic lattice signal processing," Proc. IEEE, vol. 72, no. 7, pp. 909-930, 1984.

[11] G. A. Ball, W. W. Morey, and P. K. Cheo, "Single- and multipoint fiber-laser sensors," IEEE Photon. Technol. Lett., vol. 5, no. 2, pp. 267-270, 1993.

[12] H. A. Zarem, M. E. Hoenk, W. B. Bridges, K. Vahala, and A. Yariv, "Generation of $1180 \AA$ period gratings with a Xe ion laser," Electronics Letters, vol. 24, no. 22, pp. 1366-1367, 1988.

[13] G. Recktenwald, Numerical Methods with MATLAB, Prentice Hall, Englewood Cliffs, NJ, USA, 2000.

[14] D. L. MacFarlane, J. Tong, C. Fafadia, V. Govindan, L. R. Hunt, and I. Panahi, "Extended lattice filters enabled by fourdirectional couplers," Applied Optics, vol. 43, no. 33, pp. 61246133, 2004.

L. Roberts Hunt received his B.S. degree from Baylor University in 1964 and his Ph.D. degree from Rice University in 1970, both in mathematics. He was a faculty member in the Department of Mathematics at Texas Tech University from 1969 to 1984, taking a two-year leave at the NASA Ames Research Center from 1980 to 1982. Since 1984 he has been a faculty member at the University of Texas at Dallas, where he is currently a Professor of electrical engineering. He is a Fellow of the IEEE and a licensed Professional Engineer in the State of Texas. His research interests are in nonlinear systems and control and signal processing. A previous application area was automatic flight control with the NASA Ames Research Center from 1980 to 2001. Present research projects are design of active optical lattice filters and design of power converters. 
Vishnupriya Govindan received the B.E. degree in electronics and communication engineering from the University of Madras, India, in 2002 and the M.S.E.E. degree from the University of Texas at Dallas in 2004. She is currently working towards her Ph.D. degree from the University of Utah, Salt Lake City.

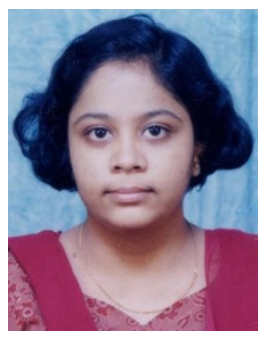

Issa Panahi received the Ph.D. degree in electrical engineering from the University of Colorado at Boulder. Dr. Panahi is currently an Assistant Professor of electrical engineering at the University of Texas at Dallas (UTD) and Director of the Statistical Signal Processing Research Laboratory. His main research interests and experience are in the areas of digital signal processing, adaptive filtering, system identifi-

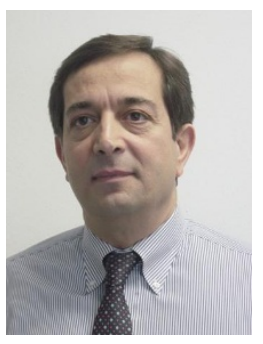
cation, detection and estimation, active noise cancellation, and embedded digital systems and processors. Dr. Panahi worked as a Research Scientist on the multidimensional and statistical signal processing at Bellaire Research Center, Shell Oil Company, Houston, for over three years before joining the DSP Division of Texas Instruments (TI) in Houston, Texas. Dr. Panahi worked at TI for over ten years as the DSP Chief Architect for TMS320C/F24xx and 28xx family of devices, Chief Technology Officer, Senior Member of the Technical Staff, and the Worldwide Applications Manager of the DSP-embedded control systems business unit. He was an Applications Manager with the Wireless OMAP Business Unit at TI before Joining UTD in 2001. Dr. Panahi holds patents and has authored/coauthored 3 DSP-based TI's books and over 35 technical papers and articles.

Jian Tong was born in China in 1976. He received the B.S. degree in electrical engineering from Shanghai Jiao Tong University, China, in 1998. From 1998 to 2001, he worked as a Technical Engineer at Lucent Technologies of Shanghai, China. Since 2001, he has been a graduate student in the University of Texas at Dallas (UTD) where he received the M.S.E.E. degree in 2003. He currently is a Ph.D. candidate and works as a Research Assistant at UTD.

Govind Kannan was born in Chennai, India. He received the B.E. degree in electronics and communication engineering from the University of Madras, India, in 2003.He is currently working towards his M.S. degree in electrical engineering at the University of Texas, Dallas, Tex. His primary research interests are in digital signal processing and filter design.
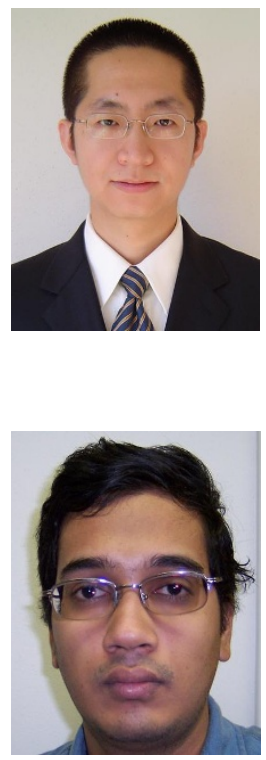

Duncan L. MacFarlane is a Full Professor of electrical engineering at the University of Texas at Dallas. Dr. MacFarlane has written more than 100 technical papers or patents in the general area of photonic systems and components. Specific research projects have included micro-optics, ultrafast lasers, photonic integrated filters, nonlinear optics, semiconductor lasers, ellipsometry, and advanced displays. Dr. MacFarlane received

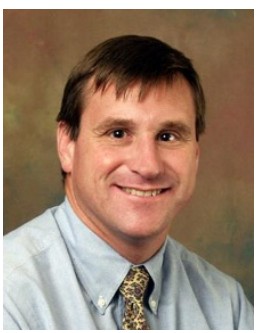
his B.S.E.E. and M.S.E.E. degrees from Brown University, his Ph.D. degree from Portland State University, and his MBA from SMU. He has worked at Schafer Associates, Texas Instruments, and at JDS Uniphase, and helped start Celion Networks, a telecommunication system start-up backed by Sequoia. He teaches graduate and undergraduate courses in electromagnetics, microwave engineering, optics, probability and statistics, and electronics, and is an Associate Dean for interdisciplinary programs. Dr. MacFarlane is a registered professional engineer in the State of Texas.

Gary Evans was born in Omak, Wash, and received the B.S.E.E. degree from the University of Washington, Seattle, in 1970 and the M.S.E.E. and Ph.D. degrees in electrical engineering and physics from the California Institute of Technology, Pasadena, in 1971 and 1975. After a postdoctoral year at Caltech, he worked for R\&D Associates, Marina Del Rey, Calif, and was a Visiting Assistant Professor in the Electrical Engineering Department at the University of Washington (1977-1979). He worked at the Aerospace Corporation, El Segundo, Calif (19791981), TRW, Redondo Beach, Calif (1981-1984), and RCA Laboratories (now Sarnoff Corporation), Princeton, NJ (1984-1992). In 1992, he joined Southern Methodist University, Dallas, Tex, as a Professor in the Electrical Engineering Department. He is a founder and member of the board of directors of Photodigm. His work on the design, growth, and fabrication of semiconductor lasers and photonic devices is described in more than 240 publications, 20 patents, and the book Surface Emitting Semiconductor Lasers Academic Press, New York, 1993. He is a licensed professional engineer and Fellow of the IEEE.

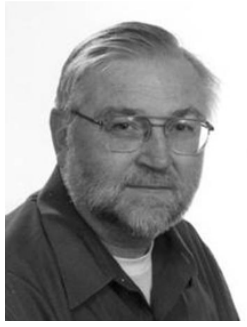

